\title{
Nonlocal gravity with worldline inversion symmetry
}

\author{
Steven Abel, ${ }^{a}$ Luca Buoninfante $^{b}$ and Anupam Mazumdar ${ }^{c}$ \\ ${ }^{a}$ Institute for Particle Physics Phenomenology, Durham University, \\ South Road, Durham, U.K. \\ ${ }^{b}$ INFN — Sezione di Napoli, Gruppo collegato di Salerno, \\ Fisciano, Salerno I-84084, Italy \\ ${ }^{c}$ Van Swinderen Institute, University of Groningen, \\ Groningen 9747 AG, The Netherlands \\ E-mail: s.a.abel@durham.ac.uk, lbuoninfante@sa.infn.it, \\ anupam.mazumdar@rug.nl
}

ABSTRACT: We construct a quadratic curvature theory of gravity whose graviton propagator around the Minkowski background respects wordline inversion symmetry, the particle approximation to modular invariance in string theory. This symmetry automatically yields a corresponding gravitational theory that is nonlocal, with the action containing infinite order differential operators. As a consequence, despite being a higher order derivative theory, it is ghost-free and has no degrees of freedom besides the massless spin-2 graviton of Einstein's general relativity. By working in the linearised regime we show that the point-like singularities that afflict the (local) Einstein's theory are smeared out.

KEYwords: String Field Theory, Models of Quantum Gravity, Spacetime Singularities, Effective Field Theories

ARXIV EPRINT: 1911.06697 


\section{Contents}

1 Introduction 1

2 Scalar propagator and worldline inversion symmetry 4

$\begin{array}{ll}2.1 & \text { Propagator in coordinate space }\end{array}$

3 Nonlocal gravitational theory $\quad 6$

$\begin{array}{lll}3.1 & \text { Graviton propagator with worldline inversion symmetry } & 8\end{array}$

4 Nonsingular gravitational potential 9

5 Conclusions 11

\section{Introduction}

Einstein's general relativity (GR) is the most widely studied theory of gravity, and its predictions have been tested to very high precision in the infrared (IR) regime, i.e. at large distances and late times [1]. Despite passing these tests, there are unsolved conceptual problems which indicate that Einstein's GR is merely an effective field theory of gravitation: it works very well at low energy but breaks down in the ultraviolet (UV). Indeed at the classical level the Einstein-Hilbert Lagrangian, $\sqrt{-g} \mathcal{R}$, suffers from the presence of blackhole and cosmological singularities [2] (implying problems in the short-distance regime), while at the quantum level it is non-renormalisable from a perturbative point of view (implying problems in the high-energy regime) [3, 4]. Therefore there is a consensus that ultimately GR will need to be extended.

One possible extension of GR is to add terms that are quadratic in curvature, such as $\mathcal{R}^{2}$ and $\mathcal{R}_{\mu \nu} \mathcal{R}^{\mu \nu}$. The resulting actions are power counting renormalisable as shown in ref. [5]. However they are still non-physical because of the presence of a massive spin2 ghost degree of freedom which classically causes Hamiltonian instabilities, and which quantum mechanically breaks the unitarity condition of the S-matrix.

The appearance of ghost modes is related to the presence of higher order time derivatives in the field equations [6]. However it is known that these unwelcome degrees of freedom can be avoided in higher derivative theories if the order of the derivatives is not finite but infinite. By introducing certain non-polynomial differential operators into the action, for example $e^{\square / \mathcal{M}^{2}}$ with $\mathcal{M}$ being a new fundamental scale, one can prevent the appearance of extra poles in the physical spectrum [7-11], because the presence of nonpolynomial derivatives makes the action nonlocal. In fact such nonlocal models were the subject of very early studies, in which it was noted that they can improve the UV behavior of loop integrals (see refs. [12-18]).

This promising property motivated deeper exploration of these nonlocal or so-called infinite derivative field theories. The first relevant applications in the gravitational context 
were made in refs. [19-23] which demonstrated the possibility of constructing a quadratic curvature theory of gravity that is classically stable and unitary at the quantum level. It has also been noted that nonlocality can regularise infinities, and many efforts have been made to resolve black hole [20, 21, 24-36, 67] and cosmological [19, 37-39] singularities. Furthermore, renormalisability [40-43], causality [44, 45], unitarity [46-50], scattering amplitudes [51-53], spontaneous breaking of symmetry [54,55] and counting of initial conditions $[56,57]$ have also been discussed and analysed. Further applications appear in the context of inflation [58-63], thermal field theory [64-66], Galilean theories [69], Casimir effect [70] and neutrino oscillation in curved space [71].

In the present work we are motivated by this kind of nonlocal field theory as an approximation to string theory. Indeed nonlocal theories have made an appearance in the context of both string field theory [72-75] and p-adic strings [76-80]. Focussing on this particular aspect, one may first ask what is the best language in which to formulate the nonlocal field theory approximation to a particular string theory? In [81, 82] it was suggested that one should most naturally be working within the worldline formalism [8387]. Indeed the immediate outcome of discarding the higher modes of a first-quantised string theory in order to get a particle approximation is precisely a worldline theory with corrections that render it nonlocal at the fundamental scale [82].

What particular nonlocal field theories might be legitimate particle approximations to string theory? There are actually two factors in a string amplitude that can be responsible for its good UV behaviour and that one might wish to imitate in a nonlocal theory: the partition function, and the world-sheet Green's function. Which one is dominant depends on the kinematics. The partition function governs the regularisation when the external momenta are low but the corresponding particle diagram would be UV divergent. For example the effective potential of non-supersymmetric (but non-tachyonic) string theories is necessarily rendered finite by the partition function. Such regularisation has been mimicked in particle theories by so-called "minimal length" theories [88]. On the other hand when the external momenta (or rather their kinematic invariants) are very large compared to the string scale it is the world-sheet Green's functions that soften the amplitudes. Their short distance behaviour is known exponentially to suppress string amplitudes even at tree level $[89,90]$.

In the case of closed strings at loop-order, modular invariance can be identified as the key element that is operating in both cases. This symmetry governs both the partition function and the Green's function. In the case of the former, modular invariance induces duality symmetries in the space-time, whose effect can be modelled by making the aforementioned "minimal-length" adjustment to the partition function [88]. Here we will instead focus on modelling the softening behaviour of the Green's function.

In ref. [81] it was suggested that theories with worldline inversion symmetry are the best way to mimick the regularising properties of modular invariance. To see why, we can begin by considering a generic two-point one-loop integral in string theory. In the "particle limit" (i.e. $\tau_{2} \gg 1$ ) it will collapse to the following heuristic form (see [82]):

$$
\mathcal{A} \sim \int_{0}^{1} d x d y \int_{0}^{1} d \tau_{1} \int_{\sim 1}^{\infty} \frac{d \tau_{2}}{\tau_{2}^{2}} \mathcal{Z}(\tau) e^{-s x(1-x) \pi \alpha^{\prime} \tau_{2}+\ldots},
$$


where $\tau=\tau_{1}+i \tau_{2}$ is the modular parameter, and $z=x+i y$ is the displacement between the two vertices. In the above the exponent is what is left of the Green's function at large $\tau_{2}$, while $s=k_{1} \cdot k_{2}$ is the kinematic invariant. ${ }^{1}$ We will ignore the accompanying prefactors because these would be the same as in the effective particle theory. In this large $\tau_{2}$ region of the fundamental domain the $y$ and $\tau_{1}$ integrals become "inert" with the latter simply enforcing the level-matching of the physical spectrum. The whole integral is then "projected" to a worldline integral over $x$ (the usual Feynman parameter) and $\pi \alpha^{\prime} \tau_{2} \equiv t$ (the usual Schwinger parameter). In other words the particle limit yields a result directly in the worldline formalism,

$$
\mathcal{A} \sim \sum_{i=\text { physical }} \int_{0}^{1} d x \int_{\sim \alpha^{\prime}}^{\infty} \frac{d t}{t} e^{-\left(s x(1-x)+m_{i}^{2}\right) t+\ldots},
$$

where the $m_{i}^{2}$ term in the exponent drops out of the partition function $\mathcal{Z}(\tau)$ and where $\sqrt{\alpha^{\prime}}$ is the string-length.

This encapsulates the effective particle theory contribution to the string amplitude. But note that invariance of the whole amplitude under the $\tau \rightarrow-1 / \tau$ modular transformation means that one could equally write the integral in the domain where it approaches the cusp at $\tau \rightarrow 0$ :

$$
\mathcal{A} \sim \sum_{i=\text { physical }} \int_{0}^{1} d y \int_{0}^{\sim \alpha^{\prime}} \frac{d t}{t} e^{-\left(s y(1-y)+m_{i}^{2}\right) \frac{1}{\mathcal{M}^{4} t}+\ldots},
$$

where we define $\mathcal{M}^{2}=1 / \pi \alpha^{\prime}$. In this limit it is $y$ rather than $x$ that drops out of the worldsheet Green's function to end up playing the role of the Feynman parameter. But since the other variable is inert, the integral as $t \rightarrow 0-$ which is a copy of $(1.2)-$ can just as well be interpreted as continuing the $t$-integral into the deep UV, but with $t \rightarrow 1 /\left(\mathcal{M}^{4} t\right)$. In [81] this was used to argued that one can capture the behaviour of the entire amplitude by writing a nonlocal theory with $t$ replaced by $T(t)=t+1 / \mathcal{M}^{4} t$ and integrating over all $t$. This approximation reproduces the asymptotic behaviour at the IR and UV cusps. It is reminiscent of string theory in the sense that the deep UV is identified as just another IR, with the difference being that in the full string theory there are an infinite number of fundamental domains not just two. In summary the gross UV/IR mixing behaviour of strings (and modular invariance) can be mimicked in the particle context by suitably modifying the Klein-Gordon propagator so that it exhibits a worldline inversion symmetry, $t \rightarrow 1 /\left(\mathcal{M}^{4} t\right)$.

In this paper we will explore such string theory inspired nonlocal field theories in the gravitational context. Our aim is to formulate a gravitational theory whose propagator around Minkowski space exhibits the above worldline inversion symmetry, and to investigate some of its consquences. This is possible despite the technicalities of writing the higher spin components of the theory in the worldline formalism [86]. The only price to pay is the introduction of nonlocality.

\footnotetext{
${ }^{1}$ Having the correct conformal weight for the vertices requires $k_{1}^{2}=k_{2}^{2}=0$ and being on shell would imply $s=0$, so we are implicitly employing the usual trick of slightly violating Lorentz invariance to retain explicit dependence on $s$.
} 
The paper is organized as follows. In section 2 we briefly review how to introduce the worldline inversion symmetry at the level of the propagator for a simple scalar field, and also show its regularising properties. In section 3 we formulate a theory of gravity with worldline inversion symmetry built in and show that such an imposition automatically requires that the Lagrangian has to be infinite order in derivatives (i.e. nonlocal) but still ghost-free. In section 4, we solve the linearised field equations in the presence of a static point-like source and explicitly show how spacetime singularities can be avoided due to the presence of nonlocality. Finally, in section 5 we draw our conclusions and discuss the outlook.

Throughout the paper we adopt the mostly positive convention for metric signature, $(-+++)$, and we work in Natural Units $\hbar=1=c$.

\section{Scalar propagator and worldline inversion symmetry}

We begin in this section by recapping and extending the scalar field Euclidean propagator, which can be defined in a general way in momentum space as an integral over a single real worldline parameter $t$ as follows [81]:

$$
\Pi\left(p^{2}\right)=\int_{0}^{\infty} d t e^{-T(t)\left(p^{2}+m^{2}\right)} .
$$

The proper-time function $T(t)$ uniquely defines the propagator in momentum space. We can immediately see that $T(t)=t$ gives the standard Schwinger parametrization for the Klein-Gordon propagator $1 /\left(p^{2}+m^{2}\right)$.

The parameter $t$ has dimensions of length-squared, and therefore modified propagators can only be characterised by a non-trivial $T(t)$ at the expense of adding a new fundamental scale. For instance, the exponential propagator that appears in string theory $[74,75]$ and infinite derivative theories $[11,19-21]$ can be recovered as

$$
T(t)=t+\frac{1}{\mathcal{M}^{2}} \Rightarrow \Pi\left(p^{2}\right)=\frac{e^{-\left(p^{2}+m^{2}\right) / \mathcal{M}^{2}}}{p^{2}+m^{2}} .
$$

Note that any modification must have a consistent IR limit, which means that the propertime must satisfy

$$
\lim _{t \rightarrow \infty} \frac{T(t)}{t}=1
$$

In addition we require the propagator to be ghost-free, i.e. we require that no negative norm states are present. A sufficient condition for this was found in ref. [81]:

$$
\operatorname{Re}\{T(t)\}>0 \quad \forall t>0 \text { and } t T\left(t^{-1}\right) \quad \text { is entire. }
$$

Following ref. [81] and the introduction, we can mimick the inversion Möbius transformation of the modular group by imposing inversion symmetry at the level of the proper-time function:

$$
t \rightarrow \frac{1}{\mathcal{M}^{4} t}
$$


where $\mathcal{M}$ is the fundamental scale required for dimensionality. It is straightforward to prove that the only proper-time function satisfying both (2.3) and the ghost-freeness condition (2.4), that is also invariant under (2.5), is

$$
T(t)=t+\frac{1}{\mathcal{M}^{\prime 2}}+\frac{1}{\mathcal{M}^{4} t},
$$

where $\mathcal{M}^{\prime}$ is a second constant parameter with dimensions of mass. We henceforth set $\mathcal{M}^{\prime} \rightarrow \infty$, so that all information on new physics is encapsulated in a single fundamental scale $\mathcal{M}$.

We are now able to compute the corresponding the scalar propagator by plugging the expression $(2.6)$ (with $1 / \mathcal{M}^{\prime 2}=0$ ) into (2.1) to find [81]:

$$
\begin{aligned}
\Pi\left(p^{2}\right) & =\frac{2}{\mathcal{M}^{2}} \mathrm{~K}_{1}\left(\frac{2\left(p^{2}+m^{2}\right)}{\mathcal{M}^{2}}\right) \equiv \frac{1}{f\left(p^{2}\right)} \frac{1}{p^{2}+m^{2}}, \\
f\left(p^{2}\right) & \equiv \frac{\mathcal{M}^{2}}{2\left(p^{2}+m^{2}\right) \mathrm{K}_{1}\left(2\left(p^{2}+m^{2}\right) / \mathcal{M}^{2}\right)},
\end{aligned}
$$

where $\mathrm{K}_{1}(z)$ is the modified Bessel function of the second kind. At low energy, $p^{2} / \mathcal{M}^{2} \ll 1$, the propagator (2.1) tends to the correct IR limit, i.e. $1 /\left(p^{2}+m^{2}\right)$, while in the high energy regime, $p^{2} / \mathcal{M}^{2} \gg 1$, it shows an exponentially suppressed behaviour (recalling that we are in Euclidean space):

$$
\Pi\left(p^{2}\right) \stackrel{\mathrm{UV}}{\longrightarrow} \frac{\sqrt{\pi} e^{-2\left(p^{2}+m^{2}\right) / \mathcal{M}^{2}}}{\sqrt{p^{2}+m^{2}}} .
$$

Note that the UV behaviour of the amplitudes is regularised through exponential suppression, in accord with the usual string picture.

Moreover, by analyzing the Bessel function in (2.7) we see that no extra pole is present besides the standard one at $p^{2}=-m^{2}$, so the propagator is ghost-free. Indeed, $\mathrm{K}_{1}(z)$ is a holomorphic function in the right-half complex plane and has a branch cut for $\operatorname{Re}\{z\}<0$ starting at $z=0$, in agreement with the structure deduced in [81].

As expected the function $f\left(p^{2}\right)$ in $(2.7)$ is non-polynomial in the momentum $p^{2}$, implying a non-polynomial differential operator in coordinate space. In fact, one can define the corresponding nonlocal action for a scalar field as

$$
S=\frac{1}{2} \int d^{4} x \phi(x) \Pi^{-1}(-\square) \phi(x),
$$

where the operator

$$
\Pi(-\square)=\frac{2}{\mathcal{M}^{2}} \mathrm{~K}_{1}\left(\frac{2\left(-\square+m^{2}\right)}{\mathcal{M}^{2}}\right)
$$

is made up of infinite order derivatives. 


\subsection{Propagator in coordinate space}

As a warm-up for the gravitational case, it is useful now to obtain the Euclidean propagator in coordinate space, and study its short-distance behaviour. This is defined as

$$
\begin{aligned}
\Pi(x) & =\int \frac{d^{4} p}{(2 \pi)^{4}} \Pi(p) e^{i p \cdot x} \\
& =\frac{2}{\mathcal{M}^{2}} \int \frac{d^{4} p}{(2 \pi)^{4}} \mathrm{~K}_{1}\left(\frac{2\left(p^{2}+m^{2}\right)}{\mathcal{M}^{2}}\right) e^{i p \cdot x} .
\end{aligned}
$$

Using polar coordinates in four dimensions, with $x=\sqrt{x^{\mu} x_{\mu}}$, we can recast the integral as

$$
\Pi(x)=\frac{1}{2 \pi^{2} \mathcal{M}^{2} x} \int_{0}^{\infty} d p p^{2} \mathrm{~K}_{1}\left(\frac{2\left(p^{2}+m^{2}\right)}{\mathcal{M}^{2}}\right) \mathrm{J}_{1}(p x),
$$

where $\mathrm{J}_{1}(x)$ is the ordinary Bessel function. The integral (2.12) cannot be performed analytically for $m \neq 0$, but it can in the massless case, which yields

$$
\Pi(x)=\frac{\mathcal{M}^{2}}{64 \pi}\left[\mathrm{I}_{0}\left(\frac{\mathcal{M}^{2} x^{2}}{8}\right)-\mathrm{L}_{0}\left(\frac{\mathcal{M}^{2} x^{2}}{8}\right)\right],
$$

where $\mathrm{I}_{0}(x)$ is a modified Bessel function of the first kind and $\mathrm{L}_{0}(x)$ is the modified Struve function. In the large distance regime, $\mathcal{M} x \gg 1$, we recover the propagator ${ }^{2}$ of the normal local theory, $1 /\left(4 \pi^{2} x^{2}\right)$, while in the short-distance regime, $\mathcal{M} x \ll 1$, the propagator is regularised as

$$
\lim _{x \rightarrow 0} \Pi(x)=\frac{\mathcal{M}^{2}}{64 \pi} .
$$

As anticipated the $1 / x^{2}$ singularity of the standard local field theory is smoothed out and regulated by the nonlocality. The same phenomenon can be observed in the massive case, by computing the $m \neq 0$ integral in eq. (2.12) numerically.

\section{$3 \quad$ Nonlocal gravitational theory}

Let us now construct an analogous gravitational theory whose propagator around the Minkowski background exhibits the same worldline inversion symmetry. In other words, we will identify the gravitational action whose linearised version gives a modified graviton propagator that has a similar structure to that in eq. (2.7) for a scalar field.

Since we aim to work with an action containing terms quadratic in the curvature tensors, let us first introduce some fundamental tools. The most general parity-invariant and torsion-free quadratic curvature action around a maximally symmetric background and up to second order variation in the metric perturbation is given by [21-23]:

$$
S=\frac{1}{2 \kappa^{2}} \int d^{4} x \sqrt{-g}\left\{\mathcal{R}+\frac{1}{2}\left[\mathcal{R} F_{1}(\square) \mathcal{R}+\mathcal{R}_{\mu \nu} F_{2}(\square) \mathcal{R}^{\mu \nu}+\mathcal{R}_{\mu \nu \rho \sigma} F_{3}(\square) \mathcal{R}^{\mu \nu \rho \sigma}\right]\right\},
$$

\footnotetext{
${ }^{2}$ In taking the asymptotic limits of the Bessel and Struve functions one encounters Stoke's phenomenon, according to which subleading contributions are discontinuous in certain regions of the complex plane. However, this does not disrupt the consistency of the IR limit which can be checked graphically.
} 
where $\kappa:=\sqrt{8 \pi G}$, with $G=1 / M_{p}^{2}$ being the Newton constant, and $F_{i}(\square)$ being two differential operators which can be uniquely determined around the Minkowski background by fixing the form of the graviton propagator [20,21].

Note that, as we are interested in second order metric perturbations around the Minkowksi metric, we are always allowed to neglect the Riemann squared term $\mathcal{R}_{\mu \nu \rho \sigma} F_{3}(\square)$ - $\mathcal{R}^{\mu \nu \rho \sigma}$ up to this order. Indeed, one can show that the following relation holds for any power $n$ of the d'Alembertian:

$$
\mathcal{R}_{\mu \nu \rho \sigma} \square^{n} \mathcal{R}^{\mu \nu \rho \sigma}=4 \mathcal{R}_{\mu \nu} \square^{n} \mathcal{R}^{\mu \nu}-\mathcal{R} \square^{n} \mathcal{R}+\mathcal{O}\left(\mathcal{R}^{3}\right)+\operatorname{div},
$$

where $\mathcal{O}\left(\mathcal{R}^{3}\right)$ stands for higher order contributions $\mathcal{O}\left(h^{3}\right)$ and div stands for total derivatives. Thus, the gravitational action in eq. (3.1) can be written as

$$
S=\frac{1}{2 \kappa^{2}} \int d^{4} x \sqrt{-g}\left\{\mathcal{R}+\frac{1}{2}\left[\mathcal{R F}_{1}(\square) \mathcal{R}+\mathcal{R}_{\mu \nu} \mathcal{F}_{2}(\square) \mathcal{R}^{\mu \nu}\right]+\mathcal{O}\left(\mathcal{R}^{3}\right)\right\},
$$

where we have defined

$$
\mathcal{F}_{1}(\square)=F_{1}(\square)-F_{3}(\square), \quad \mathcal{F}_{2}(\square)=F_{2}(\square)+4 F_{3}(\square) .
$$

By perturbing around the Minkowski metric,

$$
g_{\mu \nu}=\eta_{\mu \nu}+\kappa h_{\mu \nu}
$$

where $h_{\mu \nu}$ is the metric perturbation, we obtain the following linearised gravitational action up to order $\mathcal{O}\left(h_{\mu \nu}^{2}\right)[21]$ :

$$
\begin{aligned}
S^{(2)}=\frac{1}{4} \int d^{4} x\left\{\frac{1}{2} h_{\mu \nu} f(\square) \square h^{\mu \nu}-h_{\mu}^{\sigma} f(\square) \partial_{\sigma} \partial_{\nu} h^{\mu \nu}\right. & -\frac{1}{2} h g(\square) \square h+h g(\square) \partial_{\mu} \partial_{\nu} h^{\mu \nu} \\
& \left.+\frac{1}{2} h^{\lambda \sigma} \frac{f(\square)-g(\square)}{\square} \partial_{\lambda} \partial_{\sigma} \partial_{\mu} \partial_{\nu} h^{\mu \nu}\right\} \\
\equiv & \frac{1}{4} \int d^{4} x h_{\mu \nu} \mathcal{O}^{\mu \nu \rho \sigma} h_{\rho \sigma},
\end{aligned}
$$

with the kinetic operator defined as

$$
\begin{aligned}
\mathcal{O}^{\mu \nu \rho \sigma} \equiv \frac{1}{4}\left(\eta^{\mu \rho} \eta^{\nu \sigma}\right. & \left.+\eta^{\mu \sigma} \eta^{\nu \rho}\right) f(\square) \square-\frac{1}{2} \eta^{\mu \nu} \eta^{\rho \sigma} g(\square) \square \\
- & \frac{1}{4}\left(\eta^{\mu \rho} \partial^{\nu} \partial^{\sigma}+\eta^{\mu \sigma} \partial^{\nu} \partial^{\rho}+\eta^{\nu \rho} \partial^{\mu} \partial^{\sigma}+\eta^{\nu \sigma} \partial^{\mu} \partial^{\rho}\right) f(\square) \\
& +\frac{1}{2}\left(\eta^{\mu \nu} \partial^{\rho} \partial^{\sigma}+\eta^{\rho \sigma} \partial^{\mu} \partial^{\nu}\right) g(\square)+\frac{1}{2} \frac{f(\square)-g(\square)}{\square} \partial^{\mu} \partial^{\nu} \partial^{\rho} \partial^{\sigma} .
\end{aligned}
$$

Here $h \equiv \eta_{\mu \nu} h^{\mu \nu}$ stands for the trace and $\square=\eta^{\mu \nu} \partial_{\mu} \partial_{\nu}$ is the flat d'Alembertian operator, while the functions

$$
f(\square)=1+\frac{1}{2} \mathcal{F}_{2}(\square) \square, \quad g(\square)=1-2 \mathcal{F}_{1}(\square) \square-\frac{1}{2} \mathcal{F}_{2}(\square) \square,
$$

are combinations of the two form factors $\mathcal{F}_{i}(\square)$. 
By inverting the kinetic operator in eq. (3.6), and after having introduced a suitable gauge fixing term, one can obtain the propagator around a Minkowski background whose saturated part is given by $[7,11,20,21]$ :

$$
\Pi_{\mathrm{GR}, \mu \nu \rho \sigma}(p)=\frac{\mathcal{P}_{\mu \nu \rho \sigma}^{2}}{f(p) p^{2}}+\frac{\mathcal{P}_{s, \mu \nu \rho \sigma}^{0}}{(f(p)-3 g(p)) p^{2}},
$$

where the spin projection operators $\mathcal{P}_{\mu \nu \rho \sigma}^{2}$ and $\mathcal{P}_{s, \mu \nu \rho \sigma}^{0}$ project along the spin-2 and spin-0 components respectively, and are defined as $[91,92]$

$$
\begin{aligned}
\mathcal{P}_{\mu \nu \rho \sigma}^{2} & =\frac{1}{2}\left(\theta_{\mu \rho} \theta_{\nu \sigma}+\theta_{\mu \sigma} \theta_{\nu \rho}\right)-\frac{1}{3} \theta_{\mu \nu} \theta_{\rho \sigma}, & \mathcal{P}_{s, \mu \nu \rho \sigma}^{0} & =\frac{1}{3} \theta_{\mu \nu} \theta_{\rho \sigma}, \\
\theta_{\mu \nu} & =\eta_{\mu \nu}-\omega_{\mu \nu}, & \omega_{\mu \nu} & =\frac{k_{\mu} k_{\nu}}{k^{2}} .
\end{aligned}
$$

As a consistency check, note that for $f=1=g$ we recover the saturated part of the Einstein-Hilbert propagator [91, 92],

$$
\Pi_{\mathrm{GR}, \mu \nu \rho \sigma}(p)=\frac{\mathcal{P}_{\mu \nu \rho \sigma}^{2}}{p^{2}}-\frac{\mathcal{P}_{s, \mu \nu \rho \sigma}^{0}}{2 p^{2}}
$$

\subsection{Graviton propagator with worldline inversion symmetry}

In order to find the gravitational analogue of the propagator in eq. (2.7) we work in the simplest case in which the only on-shell propagating degrees of freedom are the massless transverse graviton of Einstein's general relativity with helicities \pm 2 . This requirement corresponds to the condition

$$
f(\square)=g(\square), \quad 2 \mathcal{F}_{1}(\square)=-\mathcal{F}_{2}(\square),
$$

which implies

$$
\Pi_{\mathrm{GR}, \mu \nu \rho \sigma}(p)=\frac{1}{f\left(p^{2}\right)}\left(\frac{\mathcal{P}_{\mu \nu \rho \sigma}^{2}}{p^{2}}-\frac{\mathcal{P}_{s, \mu \nu \rho \sigma}^{0}}{2 p^{2}}\right),
$$

whose functional form is similar to the one in (2.7): indeed we have the standard local propagator multiplied by some function $1 / f\left(p^{2}\right)$. Therefore, by choosing the function $f\left(p^{2}\right)$ as in (2.7) we obtain the following worldline inversion invariant graviton propagator:

$$
\Pi_{\mathrm{GR}, \mu \nu \rho \sigma}(p)=\frac{2}{\mathcal{M}^{2}} \mathrm{~K}_{1}\left(\frac{2 p^{2}}{\mathcal{M}^{2}}\right)\left[\mathcal{P}_{\mu \nu \rho \sigma}^{2}-\frac{1}{2} \mathcal{P}_{s, \mu \nu \rho \sigma}^{0}\right]
$$

which again is ghost-free as it possesses only one single pole at $p^{2}=0$ corresponding to the usual massless graviton degree of freedom. This procedure allows us to circumvent the technicalities of writing the higher spin components of the theory in the worldline formalism because the spin-0 part of the propagator necessarily governs the whole structure [86]. ${ }^{3}$ In

\footnotetext{
${ }^{3}$ Typically higher spins would be represented on the worldline as a global supersymmetry relevant for each diagram, with an additional supersymmetry introduced for each half-unit of spin. Even though the supersymmetry is in principle broken by the periodicity conditions of the diagram, the breaking can be considered to be a spontaneous one from a one-dimensional point of view (with the parameter $\mathrm{T}$ playing the role of a $1 \mathrm{D}$ compactification modulus if it is a one-loop diagram). Hence the tensor structure in front of any amplitude must be independent of this breaking, implying that back in the usual field theory formalism the spin-0 component of the graviton propagator then determines the structure for the higher spins.
} 
fact, by working in the usual Feynman gauge one can show that the graviton propagator reads:

$$
\begin{aligned}
\Pi_{\mathrm{GR}, \mu \nu \rho \sigma}(p) & =\left(\eta_{\mu \rho} \eta_{\nu \sigma}+\eta_{\mu \sigma} \eta_{\nu \rho}-\eta_{\mu \nu} \eta_{\rho \sigma}\right) \frac{1}{\mathcal{M}^{2}} \mathrm{~K}_{1}\left(\frac{2 p^{2}}{\mathcal{M}^{2}}\right) \\
& =\frac{1}{2}\left(\eta_{\mu \rho} \eta_{\nu \sigma}+\eta_{\mu \sigma} \eta_{\nu \rho}-\eta_{\mu \nu} \eta_{\rho \sigma}\right) \int_{0}^{\infty} d t e^{-\left(t+\frac{1}{t \mathcal{M}^{4}}\right) p^{2}}
\end{aligned}
$$

for which invariance under the wordline inversion (2.5) is now manifest; in the limit $\mathcal{M} \rightarrow \infty$ we recover the GR graviton propagator in the Feynman gauge, as expected.

From the form of the propagator, and so of the function $f(\square)$, by using the relations in eqs. (3.7), (3.11) we obtain

$$
\mathcal{F}_{1}(\square)=-\frac{1}{2} \mathcal{F}_{2}(\square)=\frac{1}{\square}+\frac{\mathcal{M}^{2}}{2 \square^{2} \mathrm{~K}_{1}\left(-\square / \mathcal{M}^{2}\right)},
$$

which gives the following gravitational action up to quadratic curvature terms:

$$
\begin{aligned}
S=\frac{1}{2 \kappa^{2}} \int d^{4} x \sqrt{-g}\left\{\mathcal{R}-G_{\mu \nu} \frac{1}{\square}\right. & \mathcal{R}^{\mu \nu} \\
& \left.\quad-\frac{\mathcal{M}^{2}}{2} G_{\mu \nu} \frac{1}{\square^{2} \mathrm{~K}_{1}\left(-\square / \mathcal{M}^{2}\right)} \mathcal{R}^{\mu \nu}\right\},
\end{aligned}
$$

where we have introduced the Einstein tensor $G_{\mu \nu}=\mathcal{R}_{\mu \nu}-1 / 2 g_{\mu \nu} \mathcal{R}$. Hence, the gravitational theory described by the action in eq. (3.16) is a higher (infinite) derivative theory of gravity which is ghost-free around a Minkowski background, despite the presence of higher order time derivatives. Up to quadratic curvature terms this is the unique action with spin-2 graviton propagator which exhibits invariance under the worldline inversion (2.5).

\section{Nonsingular gravitational potential}

In this section we wish to examine a physical implication of the nonlocality introduced by requiring the graviton propagator to be invariant under the worldline inversion symmetry in (2.5). Namely we will demonstrate that the classical linearised spacetime metric in the presence of a static point-like source for the gravitational action (3.16) is smoothed out.

To begin we compute the linearised field equation for the action (3.16), which is

$$
\begin{aligned}
& \frac{\mathcal{M}^{2}}{2 \square \mathrm{K}_{1}\left(-\square / \mathcal{M}^{2}\right)}\left(\square h_{\mu \nu}-\partial_{\sigma} \partial_{\nu} h_{\mu}^{\sigma}-\partial_{\sigma} \partial_{\mu} h_{\nu}^{\sigma}\right. \\
& \left.+\eta_{\mu \nu} \partial_{\rho} \partial_{\sigma} h^{\rho \sigma}+\partial_{\mu} \partial_{\nu} h-\eta_{\mu \nu} \square h\right)=16 \pi G T_{\mu \nu},
\end{aligned}
$$

where $T_{\mu \nu}$ is the stress-energy tensor describing the matter sector. By working in the Newtonian conformal gauge we can express the metric in isotropic coordinates as follows:

$$
d s^{2}=-(1+2 \Phi) d t^{2}+(1-2 \Phi)\left(d r^{2}+r^{2} d \Omega^{2}\right),
$$

so that $\kappa h_{00}=-2 \Phi, \kappa h_{i j}=-2 \Phi \delta_{i j}, \kappa h=-4 \Phi$, with $\Phi$ being the gravitational potential. Moreover, for a static point-like source the stress-energy tensor acquires a very simple form, 
and indeed the only non-vanishing component is the density part: $T_{\mu \nu}=m \delta_{\mu}^{0} \delta_{\nu}^{0} \delta^{(3)}(\vec{r})$, where $m$ is the mass of the object. Simplification due to staticity and spherical symmetry reveals that the only unknown, $\Phi(r)$, satisfies the following modified Poisson equation:

$$
\frac{\mathcal{M}^{2}}{2 \mathrm{~K}_{1}\left(2 \nabla^{2} / \mathcal{M}^{2}\right)} \Phi(r)=4 \pi G m \delta^{(3)}(\vec{r}) .
$$

As a consistency check, note that the local limit, $\mathcal{M} \rightarrow \infty$, recovers the standard Poisson equation whose solution is the Newtonian potential $\Phi(r)=-G m / r$.

The equation in eq. (4.3) is differential and of infinite order, which makes its solution in coordinate space quite complicated. It can be solved by Fourier transforming to momentum space through Fourier method and then transforming back, which gives

$$
\begin{aligned}
\Phi(r) & =-\frac{4 G m}{\pi \mathcal{M}^{2}} \frac{1}{r} \int_{0}^{\infty} d k k \sin (k r) \mathrm{K}_{1}\left(\frac{2 k^{2}}{\mathcal{M}^{2}}\right) \\
& =\frac{G m \mathcal{M}^{2} \pi}{16 \sqrt{2}} r\left[\mathrm{I}_{\frac{1}{4}}^{2}\left(\frac{\mathcal{M}^{2} r^{2}}{16}\right)-\mathrm{I}_{-\frac{1}{4}}^{2}\left(\frac{\mathcal{M}^{2} r^{2}}{16}\right)\right],
\end{aligned}
$$

where $\mathrm{I}_{\frac{1}{4}}(x)$ and $\mathrm{I}_{-\frac{1}{4}}(x)$ are modified Bessel functions of the first kind. By using standard relations the gravitational potential can also be recast as

$$
\Phi(r)=-\frac{G m \mathcal{M}^{2}}{16} r \mathrm{~K}_{\frac{1}{4}}\left(\frac{\mathcal{M}^{2} r^{2}}{16}\right)\left[\mathrm{I}_{\frac{1}{4}}\left(\frac{\mathcal{M}^{2} r^{2}}{16}\right)+\mathrm{I}_{-\frac{1}{4}}\left(\frac{\mathcal{M}^{2} r^{2}}{16}\right)\right] .
$$

At large distances $\mathcal{M} r \gg 1$ we recover the Newtonian behaviour as expected; while at short distances $\mathcal{M r} \ll 1$ the gravitational potential is regularised, and is entirely nonsingular at the origin:

$$
\lim _{r \rightarrow 0} \Phi(r)=-\frac{4 \pi G m \mathcal{M}}{\Gamma^{2}(-1 / 4)},
$$

where $\Gamma(x)$ is the Euler gamma function. We conclude that, as in section 2 for the singularity-free scalar, the presence of nonlocality can be instrumental in resolving the gravitational singularities that afflict standard local theories.

It is worth mentioning that, as the metric potential is monotonic with minimum at $r=0$, the linear approximation can hold true from $r=0$ all the way up to $r=\infty$ provided the following inequality is satisfied:

$$
2|\Phi|<1 \quad \Leftrightarrow \quad \frac{8 \pi G m \mathcal{M}}{\Gamma^{2}(-1 / 4)}<1
$$

In figure 1 we plot the gravitational potential (4.5) along with Newton's potential (which is singular) and with the potential corresponding to the choice (2.2) of proper-time function, which gives $\Phi_{\left(t+1 / \mathcal{M}^{2}\right)}(r)=-G m \operatorname{Erf}(r \mathcal{M} / 2) / r$ [19, 21, 30]. Note that both nonlocal potentials are strictly monotonic and are regularised at the origin with the only difference being that, in the case of worldline inversion symmetry, the spacetime metric describes a less compact gravitational system, namely $\Phi(0)<\Phi_{\left(t+1 / \mathcal{M}^{2}\right)}(0)$.

One can also check that all curvature invariants are non-singular at $r=0$, so that no curvature singularities appear at all. For instance, the Kretschmann invariant $\mathcal{R}_{\mu \nu \rho \sigma} \mathcal{R}^{\mu \nu \rho \sigma}$ 


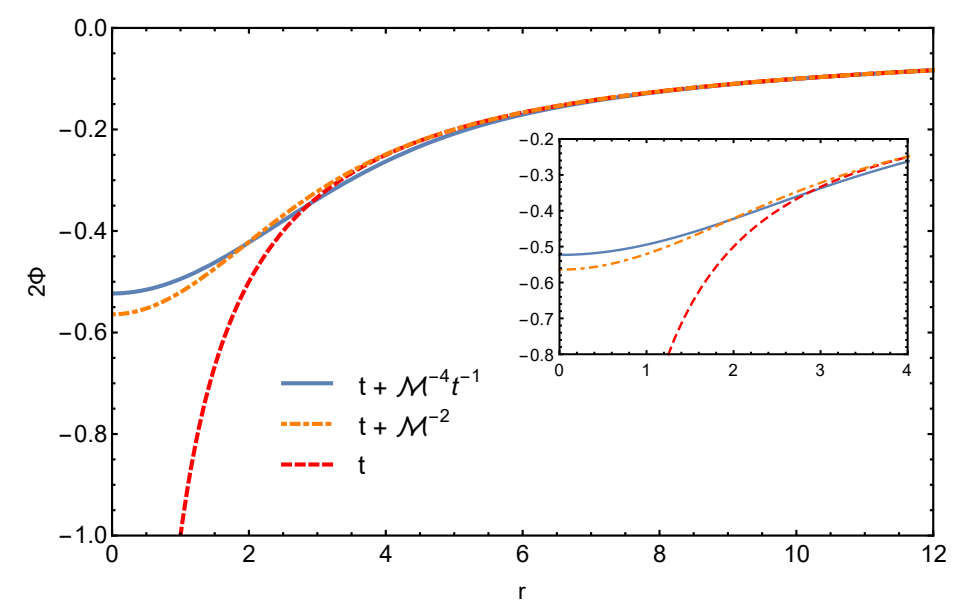

Figure 1. The nonlocal gravitational potential (blue solid line, $t+\mathcal{M}^{-4} t^{-1}$ ) generated by a point-like source in the nonlocal theory described by the action (3.16) and corresponding to the choice (2.6) with $\mathcal{M}^{\prime}=\infty$, compared to the Newtonian potential (red dashed line, $t$ ) and to the nonlocal potential corresponding to the choice (2.2) of the proper-time function (orange dotteddashed line, $t+\mathcal{M}^{-2}$ ). Here we set $G=1=\mathcal{M}$ and $m=0.5$.

for the metric in eq. (4.2), (4.5) is also finite at the origin, contrasting with the GR case in which it diverges as $1 / r^{6}$. Due to its lengthy expression we do not show it, but it is straightforward to show that the Kretschmann invariant tends to the following finite value in the short-distance regime:

$$
\lim _{r \rightarrow 0} \mathcal{R}_{\mu \nu \rho \sigma} \mathcal{R}^{\mu \nu \rho \sigma} \sim G^{2} m^{2} \mathcal{M}^{6}
$$

\section{Conclusions}

In this paper we have formulated a gravitational theory whose graviton propagator around the Minkowski background exhibits worldline inversion symmetry, which is a nonlocal particle mimicking the modular invariance of string theory. We showed that it is possible to construct such a propagator by following a quite straightforward procedure that circumvents the difficulties of writing the higher spin components of the theory in the worldline formalism [86]; see eq. (3.13). The price to pay is the introduction of non-polynomial differential operators in the gravitational action which necessarily becomes nonlocal, as shown in eq. (3.16).

Despite there being higher derivatives, such a nonlocal theory of gravity is ghost-free at tree level, and indeed the propagator possesses only one pole at $p^{2}=0$, and therefore no unhealthy degrees of freedom are present. Indeed the presence of infinite order derivatives ameliorates the short-distance behaviour of the theory entirely, and the modified gravitational potential turns out to be regularised at the origin in contrast to the Newtonian one which diverges in the limit $r \rightarrow 0$, very similar to the results in ghost free infinite derivative theories of gravity [21]. 


\section{Acknowledgments}

SAA would like to thank Nicola Dondi and Daniel Lewis for collaboration and discussions, and acknowledges support from the STFC and Royal-Society/CNRS International Cost Share Award IE160590. LB is grateful to Steven Abel for his warm hospitality at IPPP, Durham University, where this work was started. AM's research is supported by Netherlands Organization for Scientific Research (NWO) grant no. 680-91-119.

Open Access. This article is distributed under the terms of the Creative Commons Attribution License (CC-BY 4.0), which permits any use, distribution and reproduction in any medium, provided the original author(s) and source are credited.

\section{References}

[1] C.M. Will, The Confrontation between General Relativity and Experiment, Living Rev. Rel. 17 (2014) 4 [arXiv: 1403.7377] [INSPIRE].

[2] S.W. Hawking, The Large Scale Structure of Space-Time, Cambridge University Press, Cambridge U.K. (1973).

[3] G. 't Hooft and M.J.G. Veltman, One loop divergencies in the theory of gravitation, Ann. Inst. Henri Poincaré Phys. Theor. A 20 (1974) 69 [InSPIRE].

[4] M.H. Goroff and A. Sagnotti, The Ultraviolet Behavior of Einstein Gravity, Nucl. Phys. B 266 (1986) 709 [INSPIRE].

[5] K.S. Stelle, Renormalization of Higher Derivative Quantum Gravity, Phys. Rev. D 16 (1977) 953 [INSPIRE].

[6] M. Ostrogradsky, Mémoires sur les équations différentielles, relatives au problème des isopérimètres, Mem. Acad. St. Petersbourg 6 (1850) 385 [INSPIRE].

[7] N.V. Krasnikov, Nonlocal gauge theories, Theor. Math. Phys. 73 (1987) 1184 [Teor. Mat. Fiz. 73 (1987) 235] [INSPIRE].

[8] Y.V. Kuzmin, The Convergent Nonlocal Gravitation (in Russian), Sov. J. Nucl. Phys. 50 (1989) 1011 [Yad. Fiz. 50 (1989) 1630] [inSPIRE].

[9] J.W. Moffat, Finite nonlocal gauge field theory, Phys. Rev. D 41 (1990) 1177 [inSPIRE].

[10] D. Evens, J.W. Moffat, G. Kleppe and R.P. Woodard, Nonlocal regularizations of gauge theories, Phys. Rev. D 43 (1991) 499 [inSPIRE].

[11] E.T. Tomboulis, Superrenormalizable gauge and gravitational theories, hep-th/9702146 [INSPIRE].

[12] R.P. Feynman, A Relativistic cutoff for classical electrodynamics, Phys. Rev. 74 (1948) 939 [INSPIRE].

[13] A. Pais and G.E. Uhlenbeck, On Field theories with nonlocalized action, Phys. Rev. 79 (1950) 145 [INSPIRE].

[14] G.V. Efimov, Non-local quantum theory of the scalar field, Commun. Math. Phys. 5 (1967) 42 [INSPIRE].

[15] G.V. Efimov, On a class of relativistic invariant distributions, Commun. Math. Phys. 7 (1968) 138 [INSPIRE]. 
[16] V.A. Alebastrov and G.V. Efimov, A proof of the unitarity of S-matrix in a nonlocal quantum field theory, Commun. Math. Phys. 31 (1973) 1 [InSPIRE].

[17] V.A. Alebastrov and G.V. Efimov, Causality in quantum field theory with nonlocal interaction, Commun. Math. Phys. 38 (1974) 11 [INSPIRE].

[18] D.A. Kirzhnits, Nonlocal quantum field theory, Sov. Phys. Usp. 9 (1967) 692 [InSPIRE].

[19] T. Biswas, A. Mazumdar and W. Siegel, Bouncing universes in string-inspired gravity, JCAP 03 (2006) 009 [hep-th/0508194] [INSPIRE].

[20] L. Modesto, Super-renormalizable Quantum Gravity, Phys. Rev. D 86 (2012) 044005 [arXiv: 1107.2403] [INSPIRE].

[21] T. Biswas, E. Gerwick, T. Koivisto and A. Mazumdar, Towards singularity and ghost free theories of gravity, Phys. Rev. Lett. 108 (2012) 031101 [arXiv:1110.5249] [INSPIRE].

$[22]$ T. Biswas, A.S. Koshelev and A. Mazumdar, Gravitational theories with stable (anti-)de Sitter backgrounds, Fundam. Theor. Phys. 183 (2016) 97 [arXiv:1602.08475] [INSPIRE].

[23] T. Biswas, A.S. Koshelev and A. Mazumdar, Consistent higher derivative gravitational theories with stable de Sitter and anti-de Sitter backgrounds, Phys. Rev. D 95 (2017) 043533 [arXiv: 1606.01250] [INSPIRE].

[24] T. Biswas, A. Conroy, A.S. Koshelev and A. Mazumdar, Generalized ghost-free quadratic curvature gravity, Class. Quant. Grav. 31 (2014) 015022 [Erratum ibid. 31 (2014) 159501] [arXiv: 1308.2319] [INSPIRE].

[25] J. Edholm, A.S. Koshelev and A. Mazumdar, Behavior of the Newtonian potential for ghost-free gravity and singularity-free gravity, Phys. Rev. D 94 (2016) 104033 [arXiv: 1604.01989] [INSPIRE].

[26] V.P. Frolov and A. Zelnikov, Head-on collision of ultrarelativistic particles in ghost-free theories of gravity, Phys. Rev. D 93 (2016) 064048 [arXiv:1509.03336] [INSPIRE].

[27] V.P. Frolov, A. Zelnikov and T. de Paula Netto, Spherical collapse of small masses in the ghost-free gravity, JHEP 06 (2015) 107 [arXiv: 1504.00412] [INSPIRE].

[28] V.P. Frolov, Mass-gap for black hole formation in higher derivative and ghost free gravity, Phys. Rev. Lett. 115 (2015) 051102 [arXiv:1505.00492] [INSPIRE].

[29] V.P. Frolov and A. Zelnikov, Head-on collision of ultrarelativistic particles in ghost-free theories of gravity, Phys. Rev. D 93 (2016) 064048 [arXiv: 1509.03336] [INSPIRE].

[30] L. Buoninfante, A.S. Koshelev, G. Lambiase and A. Mazumdar, Classical properties of non-local, ghost- and singularity-free gravity, JCAP 09 (2018) 034 [arXiv:1802.00399] [INSPIRE].

[31] A.S. Koshelev, J. Marto and A. Mazumdar, Schwarzschild $1 / r$-singularity is not permissible in ghost free quadratic curvature infinite derivative gravity, Phys. Rev. D 98 (2018) 064023 [arXiv: 1803.00309] [INSPIRE].

[32] L. Buoninfante, A.S. Koshelev, G. Lambiase, J. Marto and A. Mazumdar, Conformally-flat, non-singular static metric in infinite derivative gravity, JCAP 06 (2018) 014 [arXiv: 1804.08195] [INSPIRE].

[33] L. Buoninfante, G. Harmsen, S. Maheshwari and A. Mazumdar, Nonsingular metric for an electrically charged point-source in ghost-free infinite derivative gravity, Phys. Rev. D 98 (2018) 084009 [arXiv: 1804.09624] [INSPIRE]. 
[34] L. Buoninfante et al., Towards nonsingular rotating compact object in ghost-free infinite derivative gravity, Phys. Rev. D 98 (2018) 084041 [arXiv: 1807.08896] [InSPIRE].

[35] E. Kilicarslan, Weak Field Limit of Infinite Derivative Gravity, Phys. Rev. D 98 (2018) 064048 [arXiv: 1808.00266] [INSPIRE].

[36] E. Kilicarslan, pp-waves as Exact Solutions to Ghost-free Infinite Derivative Gravity, Phys. Rev. D 99 (2019) 124048 [arXiv: 1903.04283] [INSPIRE].

[37] T. Biswas, T. Koivisto and A. Mazumdar, Towards a resolution of the cosmological singularity in non-local higher derivative theories of gravity, JCAP 11 (2010) 008 [arXiv: 1005.0590] [INSPIRE].

[38] T. Biswas, A.S. Koshelev, A. Mazumdar and S.Y. Vernov, Stable bounce and inflation in non-local higher derivative cosmology, JCAP 08 (2012) 024 [arXiv:1206.6374] [INSPIRE].

[39] A.S. Koshelev and S.Y. Vernov, On bouncing solutions in non-local gravity, Phys. Part. Nucl. 43 (2012) 666 [arXiv: 1202.1289] [INSPIRE].

[40] L. Modesto and L. Rachwal, Super-renormalizable and finite gravitational theories, Nucl. Phys. B 889 (2014) 228 [arXiv:1407.8036] [INSPIRE].

[41] L. Modesto and L. Rachwal, Universally finite gravitational and gauge theories, Nucl. Phys. B 900 (2015) 147 [arXiv: 1503.00261] [INSPIRE].

[42] S. Talaganis, T. Biswas and A. Mazumdar, Towards understanding the ultraviolet behavior of quantum loops in infinite-derivative theories of gravity, Class. Quant. Grav. 32 (2015) 215017 [arXiv: 1412.3467] [INSPIRE].

[43] A. Ghoshal, A. Mazumdar, N. Okada and D. Villalba, Stability of infinite derivative Abelian Higgs models, Phys. Rev. D 97 (2018) 076011 [arXiv:1709.09222] [INSPIRE].

[44] E.T. Tomboulis, Nonlocal and quasilocal field theories, Phys. Rev. D 92 (2015) 125037 [arXiv: 1507.00981] [INSPIRE].

[45] L. Buoninfante, G. Lambiase and A. Mazumdar, Ghost-free infinite derivative quantum field theory, Nucl. Phys. B 944 (2019) 114646 [arXiv: 1805. 03559] [INSPIRE].

[46] R. Pius and A. Sen, Cutkosky rules for superstring field theory, JHEP 10 (2016) 024 [Erratum JHEP 09 (2018) 122] [arXiv: 1604.01783] [INSPIRE].

[47] C.D. Carone, Unitarity and microscopic acausality in a nonlocal theory, Phys. Rev. D 95 (2017) 045009 [arXiv: 1605.02030v3] [INSPIRE].

[48] F. Briscese and L. Modesto, Cutkosky rules and perturbative unitarity in Euclidean nonlocal quantum field theories, Phys. Rev. D 99 (2019) 104043 [arXiv: 1803.08827] [InSPIRE].

[49] P. Chin and E.T. Tomboulis, Nonlocal vertices and analyticity: Landau equations and general Cutkosky rule, JHEP 06 (2018) 014 [arXiv: 1803.08899] [INSPIRE].

[50] M. Christodoulou and L. Modesto, Note on Reflection Positivity in Nonlocal Gravity, JETP Lett. 109 (2019) 286 [arXiv:1803. 08843] [INSPIRE].

[51] T. Biswas and N. Okada, Towards LHC physics with nonlocal Standard Model, Nucl. Phys. B 898 (2015) 113 [arXiv:1407.3331] [INSPIRE].

[52] P. Donà, S. Giaccari, L. Modesto, L. Rachwal and Y. Zhu, Scattering amplitudes in super-renormalizable gravity, JHEP 08 (2015) 038 [arXiv: 1506.04589] [INSPIRE].

[53] L. Buoninfante, A. Ghoshal, G. Lambiase and A. Mazumdar, Transmutation of nonlocal scale in infinite derivative field theories, Phys. Rev. D 99 (2019) 044032 [arXiv:1812.01441] [INSPIRE]. 
[54] F.S. Gama, J.R. Nascimento, A.Y. Petrov and P.J. Porfirio, Spontaneous Symmetry Breaking in the Nonlocal Scalar QED, arXiv:1804.04456 [INSPIRE].

[55] M.N. Hashi, H. Isono, T. Noumi, G. Shiu and P. Soler, Higgs Mechanism in Nonlocal Field Theories, JHEP 08 (2018) 064 [arXiv:1805.02676] [INSPIRE].

[56] N. Barnaby and N. Kamran, Dynamics with infinitely many derivatives: The Initial value problem, JHEP 02 (2008) 008 [arXiv:0709.3968] [INSPIRE].

[57] G. Calcagni, L. Modesto and G. Nardelli, Initial conditions and degrees of freedom of non-local gravity, JHEP 05 (2018) 087 [Erratum JHEP 05 (2019) 095] [arXiv:1803.00561] [INSPIRE].

[58] N. Barnaby, T. Biswas and J.M. Cline, p-adic Inflation, JHEP 04 (2007) 056 [hep-th/0612230] [INSPIRE].

[59] T. Biswas, R. Brandenberger, A. Mazumdar and W. Siegel, Non-perturbative Gravity, Hagedorn Bounce $\& 3$ CMB, JCAP 12 (2007) 011 [hep-th/0610274] [INSPIRE].

[60] T. Biswas and A. Mazumdar, Super-Inflation, Non-Singular Bounce and Low Multipoles, Class. Quant. Grav. 31 (2014) 025019 [arXiv: 1304.3648] [INSPIRE].

[61] A.S. Koshelev, L. Modesto, L. Rachwal and A.A. Starobinsky, Occurrence of exact $R^{2}$ inflation in non-local UV-complete gravity, JHEP 11 (2016) 067 [arXiv:1604.03127] [INSPIRE].

[62] A.S. Koshelev, K. Sravan Kumar and A.A. Starobinsky, $R^{2}$ inflation to probe non-perturbative quantum gravity, JHEP 03 (2018) 071 [arXiv:1711.08864] [INSPIRE].

[63] K. Sravan Kumar and L. Modesto, Non-local Starobinsky inflation in the light of future $C M B$, arXiv: 1810.02345 [INSPIRE].

[64] T. Biswas, J.A.R. Cembranos and J.I. Kapusta, Thermal Duality and Hagedorn Transition from p-adic Strings, Phys. Rev. Lett. 104 (2010) 021601 [arXiv:0910.2274] [INSPIRE].

[65] T. Biswas, J.A.R. Cembranos and J.I. Kapusta, Thermodynamics and Cosmological Constant of Non-Local Field Theories from p-Adic Strings, JHEP 10 (2010) 048 [arXiv:1005.0430] [INSPIRE].

[66] T. Biswas, J.A.R. Cembranos and J.I. Kapusta, Finite Temperature Solitons in Non-Local Field Theories from p-Adic Strings, Phys. Rev. D 82 (2010) 085028 [arXiv:1006.4098] [INSPIRE].

[67] L. Buoninfante and A. Mazumdar, Nonlocal star as a blackhole mimicker, Phys. Rev. D 100 (2019) 024031 [arXiv: 1903. 01542] [inSPIRE].

[68] L. Buoninfante, A. Mazumdar and J. Peng, Nonlocality amplifies echoes, Phys. Rev. D 100 (2019) 104059 [arXiv:1906.03624] [INSPIRE].

[69] L. Buoninfante, G. Lambiase and M. Yamaguchi, Nonlocal generalization of Galilean theories and gravity, Phys. Rev. D 100 (2019) 026019 [arXiv:1812.10105] [INSPIRE].

[70] L. Buoninfante, G. Lambiase, L. Petruzziello and A. Stabile, Casimir effect in quadratic theories of gravity, Eur. Phys. J. C 79 (2019) 41 [arXiv:1811.12261] [inSPIRE].

[71] L. Buoninfante, G.G. Luciano, L. Petruzziello and L. Smaldone, Neutrino oscillations in extended theories of gravity, arXiv:1906.03131 [INSPIRE].

[72] E. Witten, Noncommutative Geometry and String Field Theory, Nucl. Phys. B 268 (1986) 253 [INSPIRE]. 
[73] D.A. Eliezer and R.P. Woodard, The Problem of Nonlocality in String Theory, Nucl. Phys. B 325 (1989) 389 [INSPIRE].

[74] A.A. Tseytlin, On singularities of spherically symmetric backgrounds in string theory, Phys. Lett. B 363 (1995) 223 [hep-th/9509050] [INSPIRE].

[75] W. Siegel, Stringy gravity at short distances, hep-th/0309093 [INSPIRE].

[76] P.G.O. Freund and M. Olson, Nonarchimedean Strings, Phys. Lett. B 199 (1987) 186 [INSPIRE].

[77] L. Brekke, P.G.O. Freund, M. Olson and E. Witten, Nonarchimedean String Dynamics, Nucl. Phys. B 302 (1988) 365 [INSPIRE].

[78] P.G.O. Freund and E. Witten, Adelic String Amplitudes, Phys. Lett. B 199 (1987) 191 [INSPIRE].

[79] P.H. Frampton and Y. Okada, Effective Scalar Field Theory of p-adic String, Phys. Rev. D 37 (1988) 3077 [INSPIRE].

[80] B. Dragovich, A.Y. Khrennikov, S.V. Kozyrev and I.V. Volovich, On p-Adic Mathematical Physics, Anal. Appl. 1 (2009) 1 [arXiv:0904.4205] [INSPIRE].

[81] S. Abel and N.A. Dondi, UV Completion on the Worldline, JHEP 07 (2019) 090 [arXiv: 1905.04258] [INSPIRE].

[82] S. Abel and D. Lewis, in preparation.

[83] R.P. Feynman, Mathematical formulation of the quantum theory of electromagnetic interaction, Phys. Rev. 80 (1950) 440 [InSPIRE].

[84] M.J. Strassler, Field theory without Feynman diagrams: One loop effective actions, Nucl. Phys. B 385 (1992) 145 [hep-ph/9205205] [INSPIRE].

[85] M.G. Schmidt and C. Schubert, Worldline Green functions for multiloop diagrams, Phys. Lett. B 331 (1994) 69 [hep-th/9403158] [INSPIRE].

[86] C. Schubert, Perturbative quantum field theory in the string inspired formalism, Phys. Rept. 355 (2001) 73 [hep-th/0101036] [INSPIRE].

[87] C. Schubert, Lectures on the Worldline Formalism, talk given at the School on Spinning Particles in Quantum Field Theory: Worldline Formalism, Higher Spins and Conformal Geometry, Morelia, Michoacán, Mexico, 19-23 November 2012 and online at https://indico.cern.ch/event/206621/attachments/317309.

[88] T. Padmanabhan, Duality and zero point length of space-time, Phys. Rev. Lett. 78 (1997) 1854 [hep-th/9608182] [INSPIRE].

[89] D.J. Gross and P.F. Mende, The High-Energy Behavior of String Scattering Amplitudes, Phys. Lett. B 197 (1987) 129 [INSPIRE].

[90] D.J. Gross and P.F. Mende, String Theory Beyond the Planck Scale, Nucl. Phys. B 303 (1988) 407 [INSPIRE].

[91] P. Van Nieuwenhuizen, On ghost-free tensor lagrangians and linearized gravitation, Nucl. Phys. B 60 (1973) 478 [inSPIRE].

[92] T. Biswas, T. Koivisto and A. Mazumdar, Nonlocal theories of gravity: the flat space propagator, in proceedings of the Barcelona Postgrad Encounters on Fundamental Physics, Barcelona, Spain, 17-19 October 2012, pp. 13-24 [arXiv:1302.0532] [INSPIRE]. 